DOI: https://doi.org/10.11144/Javeriana.umed61-4.tepb

\title{
La importancia de la presunción clínica en el diagnóstico de tromboembolismo pulmonar de baja probabilidad: presentación de caso
}

The Importance of Clinical Presumption in the Diagnosis of Low Probability PE: Case Report

Recibido: 20 Marzo 2020 | Aceptado: 04 Junio 2020

\author{
Gustavo Andrés Flórez González \\ Médico residente de Medicina de Urgencias, Pontificia \\ Universidad Javeriana, Bogotá, Colombia \\ Andrea del Pilar Acevedo Guiot \\ Médico especialista en Medicina de Urgencias, \\ Pontificia Universidad Javeriana, Bogotá, Colombia
}

a Autor de correspondencia: florez.gustavo@javeriana.edu.co

Cómo citar: Flórez González GA, Acevedo Guiot AP. La importancia de la presunción clínica en el diagnóstico de TEP de baja probabilidad. Univ. Med. 2020;61(4). https://doi.org/10.11144/Javeriana.umed 61-4.tepb

\section{RESUMEN}

El tromboembolismo pulmonar es una condición clínica ligada a diversos factores de riesgo y a una alta morbimortalidad. Las escalas orientadas en establecer una probabilidad pretest están diseñadas para detectar factores de riesgo de presentar la enfermedad; sin embargo, su diagnóstico es un reto cuando no existen dichos factores, como en la presentación de este caso, atendido en un hospital de cuarto nivel en Bogotá (Colombia). Se tomó como base la guía de tromboembolismo pulmonar de la Sociedad Europea de Cardiología de 2019 y se realizó una búsqueda de la literatura utilizando los términos MeSH: tromboembolismo pulmonar en pacientes de baja probabilidad en las fuentes Pubmed, Google Schoolar y Scopus, con obtención de resultados insuficientes. Ello hace importante reportar este caso y conlleva resaltar el impacto de la presunción clínica como herramienta fundamental de diagnóstico en urgencias.

Palabras clave

embolia pulmonar; infarto pulmonar; dolor en el pecho.

\section{ABSTRACT}

Pulmonary thromboembolism is a clinical condition linked to various risk factors and high morbidity. The scales aimed at establishing a pretest probability are designed to detect risk factors for presenting the disease, however their diagnosis is a challenge when there are no such factors, as in the report of this case attended in a fourth-level hospital in Bogotá (Colombia). The 2019 European Society of Cardiology Pulmonary Thromboembolism guide was based on the pulmonary thromboembolism guide and a search was conducted using the terms $\mathrm{MeSH}$ : pulmonary thromboembolism in low probability patients in Pubmed, Google Schoolar, and Scopus sources, with insufficient results which makes it important to report this case and leads to highlighting the impact of clinical presumption as a fundamental tool for diagnosis in the emergency.

Keywords

pulmonary embolism; pulmonary infarction; chest pain. 


\section{Introducción}

El tromboembolismo pulmonar es una condición clínica en la cual se produce una oclusión arterial por efecto de un trombo que se desplaza desde el lecho venoso de las extremidades inferiores, la mayoría de veces y en menos del $10 \%$ de los casos provenientes de las extremidades superiores (1), que viaja por el torrente sanguíneo, hasta alojarse finalmente en el lecho arterial pulmonar con el consecuente efecto mecánico e inflamatorio derivado de la acción plaquetaria que conlleva la producción de tromboxano A2, serotonina, endotelina-1, prostaglandinas F2 $\alpha, \mathrm{H} 2, \mathrm{D} 2$, E2 y leucotrieno D4 (2). Ello contribuye a modificar el intercambio gaseoso en los pulmones y en los casos más severos puede dar lugar a una disfunción cardiaca como parte de la afectación vascular (3), lo cual puede conducir a una presentación clínica variada, consistente en dolor torácico, disnea, tos, taquipnea, taquicardia, agregados pulmonares, hemoptisis, síncope y choque $(4,5)$.

El embolismo venoso, como parte del proceso para la generación del tromboembolismo pulmonar, es más frecuente conforme aumenta la edad (1) y es más frecuente en mujeres que en hombres cuando se comparan por debajo de los 55 años (6). Entre el $20 \%$ y $30 \%$ de los casos no se identifica causa alguna (1).

La incidencia de la embolia pulmonar es de 29-78 personas por cada 10.000 (7) y su mortalidad oscila entre el 19,4 y 32,3 por cada 100.000 personas (8). Diversos factores pueden condicionar su presentación, como cirugía mayor, trastornos de hipercoagulabilidad, estancia hospitalaria por condiciones médicas agudas, confinamiento en cualquier unidad de cuidado, trauma, fracturas, cáncer activo, enfermedad neurológica que limite la marcha, embarazo, posparto, anticonceptivos orales y uso de estrógenos (7).

$\mathrm{Su}$ diagnóstico presuntivo se establece a partir la presentación clínica y escalas de probabilidad pretest, y de acuerdo con ello se define la conducta más apropiada en términos de estudios complementarios. Como alternativas están el estudio con dímero D, la angiotomografía de tórax, la gammagrafía ventilación/perfusión, el ecocardiograma y el examen Doppler de miembros inferiores (9).

Para tal fin, y con el objetivo de optimizar recursos y disminuir la exposición a estudios innecesarios, se han diseñado diferentes escalas que buscan orientar su estudio hacia la metodología más apropiada.

Dentro de las escalas validadas con mayor uso se encuentran la Escala de Wells, la Escala de Geneva y sus respectivas formas simplificadas, que clasifican a los pacientes en baja, intermedia y alta probabilidad pretest, sin que se hayan documentado diferencias en sus rendimientos (10). Sin embargo, es importante tener en cuenta que dichas reglas son tan solo un apoyo a la hora de la toma de decisiones, dado que hasta el momento ninguna de estas escalas de predicción es superior al juicio clínico (11).

El hacer uso de estas reglas y hablar de una probabilidad baja o intermedia para tromboembolismo pulmonar conlleva la realización de dímero $\mathrm{D}$, el cual permitirá definir la necesidad de un estudio complementario con angiotomografía de tórax o gammagrafía pulmonar, según el contexto clínico del paciente o la disponibilidad de recursos en cada institución.

Adicionalmente, se diseñaron las escalas Pulmonary Embolism Rule-Out Criteria (PERC) y la canadiense, con el objetivo de determinar la viabilidad de dar egreso a pacientes con baja probabilidad para tromboembolismo pulmonar sin necesidad de realizar estudio con dímero $D$ (12). La escala PERC cuenta con una sensibilidad del $96 \%$ y con una especificidad del $27 \%$; entre tanto, la escala canadiense cuenta con una sensibilidad y una especificidad del $78 \%$ y $44 \%$, respectivamente (13).

Sin embargo, un pequeño grupo de pacientes $(6,1 \%)$ no presenta los factores de riesgo descritos en dichas escalas, en los cuales el diagnóstico se realiza por sospecha clínica o incluso de forma incidental (13), aun cuando el dolor torácico pleurítico es la manifestación clínica más encontrada en aquellos pacientes con baja probabilidad de tromboembolismo pulmonar (14), por lo cual presentamos el siguiente caso. 


\section{Caso clínico}

A continuación, se publican los datos de la historia clínica, al igual que los soportes de los exámenes paraclínicos realizados previo consentimiento informado del paciente y se declara que no se tiene ningún conflicto de intereses en el diagnóstico o tratamiento que se le realizó; únicamente se describen los sucesos de manera cronológica y exacta.

El caso corresponde a un hombre de 22 años de edad, quien consultó a la 1:45 a. m. por un cuadro clínico de tres horas de evolución, consistente en dolor torácico intenso de presentación súbita que se exacerbaba con la inspiración profunda sin otros síntomas asociados y antecedentes negativos en el interrogatorio.

En el examen físico presentó una tensión arterial de 120/69, una frecuencia cardiaca de 84 latidos por minuto, una frecuencia respiratoria de 20 respiraciones por minuto, una saturación del $95 \%$ y una temperatura $36,7^{\circ} \mathrm{C}$.

La mucosa oral estaba húmeda, el cuello no tenía masas ni ingurgitación yugular, el tórax era simétrico y los ruidos cardiacos eran rítmicos, sin agregados a la auscultación cardiopulmonar. El abdomen no mostraba cambios, las extremidades estaban sin edemas, los pulsos estaban conservados y los neurológicos sin déficit.

Dadas las características del cuadro clínico, se descartaron como posibles etiologías neumotórax, síndrome aórtico y pericarditis. Por ello, se solicitó la toma de un electrocardiograma (figura 1) y de una radiografía de tórax (figura 2) como estudios iniciales, sin que estos evidenciaran cambios en la repolarización cardiaca o hallazgos compatibles con las sospechas iniciales; sin embargo, ante la persistencia de dolor durante la revaloración a las 6:50 a.m., a pesar del manejo analgésico, se plantearon otros posibles diagnósticos como miocarditis y tromboembolismo pulmonar de baja probabilidad, con Wells 0 (tabla 1) (15), y se solicitó dímero D, a pesar de un PERC de 0 (tabla 2) (16), con obtención de un valor en $3880 \mathrm{ng} / \mathrm{ml}$. Adicionalmente, troponina I en 0 y hemograma con leucocitos:
10.200; hemoglobina: 16,3; plaquetas: 252.900; neutrófilos: 6900; eosinófilos: 0; basófilos: 0.

\section{Figura 1}

Electrocardiograma

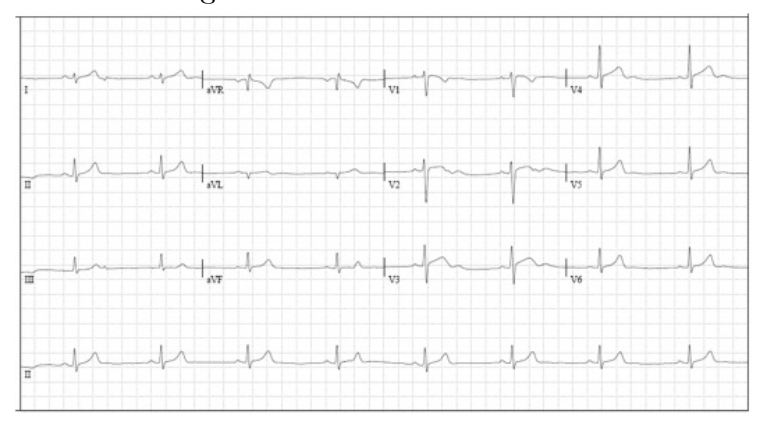

Figura 2.

Radiografía de tórax

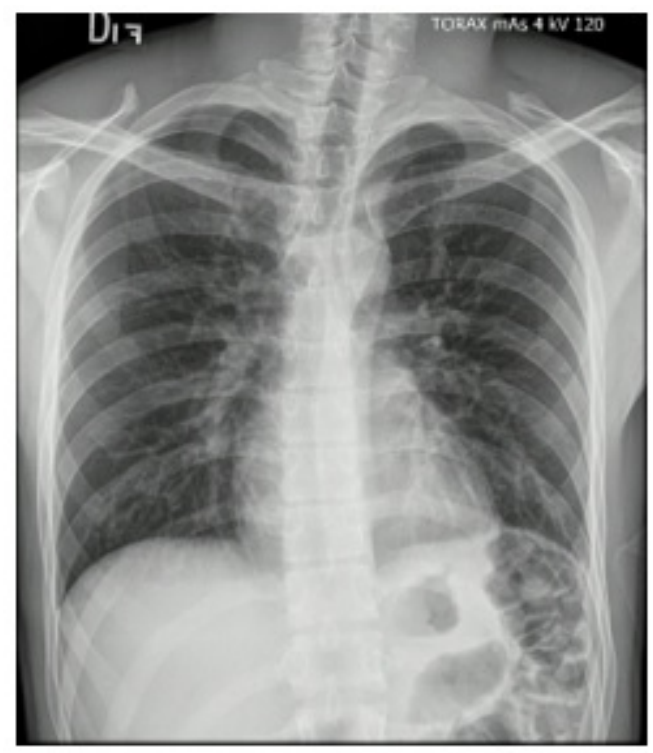

Tabla 1

Escala de Wells

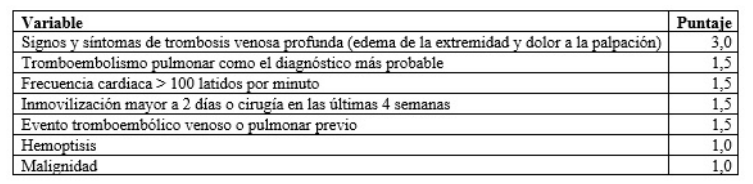

Tabla 2

Escala PERC

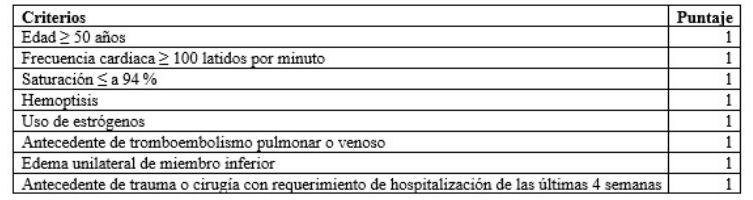


Posteriormente, se solicitó un estudio con angiotomografía de tórax (figuras 3 y 4). Los resultados se obtuvieron a las 11:50 a. m. con reporte de opacidades de las ramas segmentarias y subsegmentarias del lóbulo inferior izquierdo, que confirmaron la sospecha de tromboembolismo pulmonar. Se inició manejo anticoagulante con enoxaparina y un día posterior a su ingreso se decidió dar manejo ambulatorio con rivaroxabán y orden de estudios complementarios para hipercoagulabilidad.

\section{Figura 3}

Angiotomografía de tórax vista 1

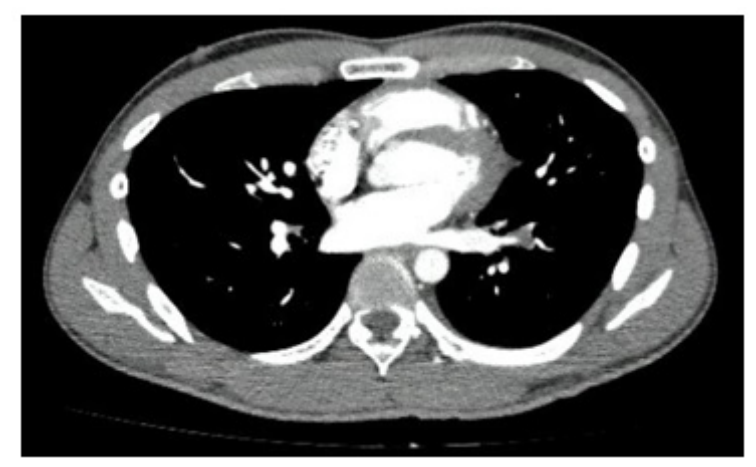

Figura 4.

Angiotomografía de tórax, vista 2

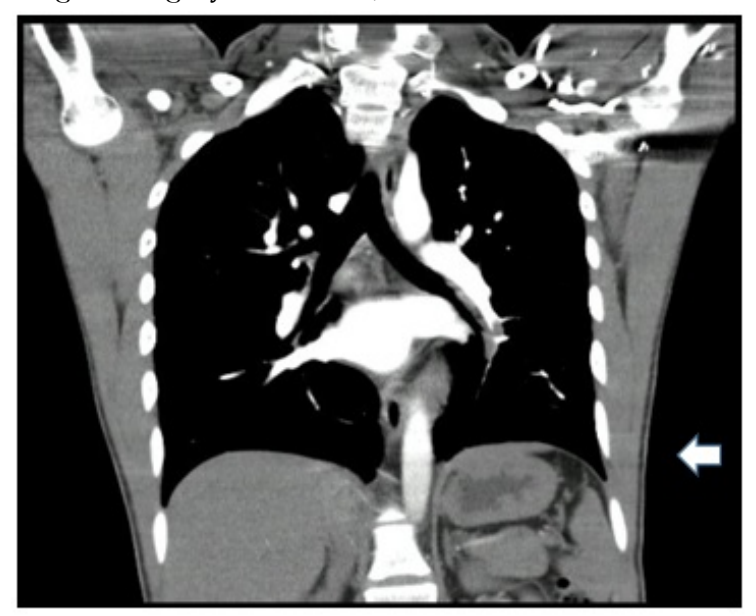

\section{Discusión}

En la actualidad, se dispone de diferentes escalas que realizan aproximaciones diagnósticas al tromboembolismo pulmonar y que tratan de optimizar recursos utilizando la metodología más adecuada; sin embargo, el juicio clínico continúa siendo un elemento fundamental a la hora de tomar decisiones, dado que las escalas no están exentas de error, y en el caso de aquellos pacientes de bajo riesgo, la escala PERC no incluye el dolor torácico pleurítico como parte de sus criterios, a pesar de ser una manifestación frecuente - se encuentra en el $56 \%$ de los pacientes con tromboembolismo pulmonar que cursan con PERC falsamente negativo, como lo mostró el estudio Multicenter Emergency Medicine Pulmonary Embolism in the Real World Registry(17). Por ende, dicha manifestación debería ser una condición para tener en cuenta cuando surge el tromboembolismo pulmonar como posible diagnóstico, pese a no existir otras asociaciones en la presentación clínica (tabla 3) (17).

\section{Tabla 3}

Frecuencia sensibilidad y especificidad de signos y sintomas en tromboembolismo pulmonar

\begin{tabular}{|c|c|c|c|}
\hline Síntomas & Frecuencia (\%) & S (\%) & E (\%) \\
\hline Disnea & 73 & 36 & 25 \\
\hline Dolor torácico pleurítico & 66 & 41 & 53 \\
\hline Tos & 37 & 33 & 28 \\
\hline Hemoptisis & 13 & 18 & 22 \\
\hline Signos & Frecuencia (\%) & $\mathrm{S}(\%)$ & E (\%) \\
\hline Taquipnea ( $>19$ por minuto) & 70 & 53 & 42 \\
\hline $\begin{array}{l}\text { Taquicardia ( }>100 \text { por } \\
\text { minuto) }\end{array}$ & 51 & 24 & 14 \\
\hline Agregados pulmonares & 30 & 17 & 14 \\
\hline $\begin{array}{l}\text { Aumento en el componente } \\
\text { pulmonar del segundo ruido }\end{array}$ & 23 & 11 & 3 \\
\hline
\end{tabular}

La relevancia de este caso clínico es la presentación de una entidad potencialmente fatal en un hombre joven sin factores de riesgo en la historia de ingreso a urgencias, con dolor torácico pleurítico como única manifestación en quien, según el protocolo, habría que descartar otras entidades como principales causas del dolor. Además, había ingresado con una adecuada saturación de oxígeno, sin taquicardia y sin hallazgos objetivos en los exámenes iniciales, como el electrocardiograma o la radiografía de tórax, sugestivos de entidad infecciosa, traumática o vascular. Por ello, a pesar de no cumplir con los criterios que indicaban la toma de un examen complementario como dímero $\mathrm{D}$, se decide su realización por sospecha clínica, dada la intensidad y persistencia del dolor. Se encontró positivo, por lo cual se tomó la angiotomografía, 
que confirmó el diagnóstico de tromboembolismo pulmonar.

Basándonos en los datos, obtenidos de la estadística del Hospital Universitario San Ignacio, institución de cuarto nivel en Bogotá (Colombia), para septiembre de 2019 (mes en que se atendió nuestro caso), se trataron un número aproximado de 1617 personas por mes, y de estas alrededor de 213 tenían como motivo de consulta el dolor torácico (13,2\%). Teniendo en cuenta el impacto en morbimortalidad de las patologías asociadas a dicho motivo de consulta, es un reto para todo el equipo de salud hacer una aproximación rápida, verídica y eficaz.

En un contexto de inestabilidad hemodinámica, múltiples factores de riesgo, comorbilidades o exámenes de ingreso como electrocardiogramas anormales, el diagnóstico se da casi por inercia; sin embargo, el desafío está en detectar enfermedades potencialmente fatales en aquellos pacientes de baja probabilidad para tromboembolismo pulmonar, con síntomas y signos con baja sensibilidad y especificidad para determinadas enfermedades. Ahí es cuando las escalas o las unidades de medida empiezan a perder el peso y cuando la semiología y el criterio del clínico comienzan a tomar validez e importancia. Por lo tanto, el uso de estas escalas se convierte en una herramienta de gran ayuda que deberá siempre estar integrada al juicio clínico, para favorecer la toma de decisiones y proporcionar la mejor intervención al paciente.

\section{Referencias}

1. Wendelboe AM, Raskob GE. Global burden of thrombosis: epidemiologic aspects. Circ Res. 2016;118(9):1340-7.

2. Utsonomiya $\mathrm{T}$, Krausz MM, Levine L, Shepro D, Hechtman HB. Thromboxane mediation of cardiopulmonary effects of embolism. J Clin Invest. 1982;70(2):361-8.

3. Smulders YM. Pathophysiology and treatment of haemodynamic instability in acute pulmonary embolism: the pivotal role of pulmonary vasoconstriction. Cardiovasc Res. 2000;48(1):23-33.

4. Benotti JR, Dalen JE. The natural history of pulmonary embolism. Clin Chest Med. 1984;5(3):403-10.

5. Stein PD, Beemath A, Matta F, Weg JG, Yusen RD, Hales CA, et al. Clinical characteristics of patients with acute pulmonary embolism: data from PIOPED II. Am J Med. 2007;120(10):871-9.

6. Engbers MJ, van Hylckama Vlieg A, Rosendaal FR. Venous thrombosis in the elderly: Incidence, risk factors and risk groups. J Thromb Haemost. 2010;8(10):2105-12.

7. Heit JA, Spencer FA, White RH. The epidemiology of venous thromboembolism. J Thromb Thrombolysis. 2016;41(1):3-14.

8. Anderson FA, Spencer FA. Risk factors for venous thromboembolism. Circulation. 2003;107(90231):9I-16. h ttps://doi.org/10.1161/01.CIR.0000078 469.07362.E6

9. Konstantinides SV, Meyer G, Becattini C, Bueno H, Geersing G-J, Harjola VP, et al. 2019 ESC Guidelines for the diagnosis and management of acute pulmonary embolism developed in collaboration with the European Respiratory Society (ERS) : The Task Force for the diagnosis and management of acute pulmonary embolism of the European Society of Cardiology (ESC). Eur Heart J. 2019;54(3):1901647. https://doi.org/1 0.1183/13993003.01647-2019

10. Douma RA, Mos ICM, Erkens PMG, Nizet TAC, Durian MF, Hovens MM, et al. Performance of 4 clinical decision rules in the diagnostic management of acute pulmonary embolism. Ann Intern Med. 2011;154(11):709-18. https://doi.org/1 
$0.7326 / 0003-4819-154-11-201106070$ $-00002$

11. Sanders S, Doust J, Glasziou P. A systematic review of studies comparing diagnostic clinical prediction rules with clinical judgment. PLoS One. 2015;10(6):1-25. http://dx.doi.org/10. 1371/journal.pone.0128233

12. Freund Y, Cachanado M, Aubry A, Orsini C, Raynal PA, FéralPierssens AL, et al. Effect of the pulmonary embolism rule-out criteria on subsequent thromboembolic events among low-risk emergency department patients the PROPER randomized clinical trial. JAMA. 2018;319(6):559-66. https://doi.org/10 $.1001 /$ jama.2017.21904

13. Kline JA, Mitchell AM, Kabrhel C, Richman PB, Courtney DM. Clinical criteria to prevent unnecessary diagnostic testing in emergency department patients with suspected pulmonary embolism. J Thromb Haemost. 2004;2 (8):1247-55.

14. Kline JA, Slattery D, O'Neil BJ, Thompson JR, Miller CD, Schreiber D, et al. Clinical features of patients with pulmonary embolism and a negative PERC rule result. Ann Emerg Med. 2013;61(1):122-4. http://dx.doi.org/10 .1016/j.annemergmed.2012.06.494

15. Van Belle A, Büller $H$, Huisman M, Huisman P, Kaasjaer $\mathrm{K}$, Kamphuisen P. Effectiveness of managing suspected pulmonary embolism using an algorithm. JAMA. 2006;295(2):172-9. https://doi.org/10. 1001/jama.295.2.172

16. Kline JA, Courtney DM, Kabrhel C, Moore CL, Smithline HA, Plewa $\mathrm{MC}$, et al. Prospective multicenter evaluation of the pulmonary embolism rule-out criteria. J Thromb Haemost. 2008;6(5):772-80.
17. Pollack CV, Schreiber D, Goldhaber SZ, Slattery D, Fanikos J, O'Neil BJ, et al. Clinical characteristics, management, and outcomes of patients diagnosed with acute pulmonary embolism in the emergency department: Initial report of EMPEROR (multicenter emergency medicine pulmonary embolism in the real world registry). J Am Coll Cardiol. 2011;57 (6):700-6. http://dx.doi.org/10 $.1016 /$ j.jacc.2010.05.071

\section{Notas}

Conflicto de intereses: Ninguno. 